\title{
On the higher-order effects in target single ionization by bare ions in the perturbative regime
}

\author{
A.B. Voitkiv, B.Najjari and J.Ullrich \\ Max-Planck-Institut für Kernphysik, Saupfercheckweg 1, D-69117 Heidelberg, Germany
}

(April 9, 2003)

\begin{abstract}
We consider hydrogen and helium ionization with emission of soft electrons in high-velocity collisions with bare ions in the perturbative regime $\left|Z_{p}\right| / v_{p} \lesssim 0.1$, where $Z_{p}$ is the projectile charge and $v_{p}$ the collision velocity. For such collisions it is usually assumed that the first order approximation in the projectile-target interaction yields good results for single ionization. However, by performing calculations in the first and second Born, Glauber and CDW-EIS approximations, we show that higher-order effects can considerably influence electron emission already in the collision plane where the main part of the emission occurs. Moreover, the deviations from the first order results become even stronger if the electron emission is analysed in the plane perpendicular to the momentum transfer. In this plane a pronounced structure appears in the fully differential cross section. This structure is different for collisions with $Z_{p}>0$ and $Z_{p}<0$ and the difference remains noticeable even for collisions with protons and antiprotons moving at velocities approaching the speed of light. It is also found that, on average, the higher-order effects are relatively more important for collisions with negatively charged projectiles. The deviations from first order results for emission from hydrogen in the perturbative regime are attributed mainly to the projectile interaction with the hydrogen nucleus. In case of helium single ionization our calculations suggest that a proper description of electron emission in the perpendicular plane may be very demanding with respect to the quality of the approximations for the initial and final helium states.
\end{abstract}

PACS:34.10.+x, 34.50.-s, 34.50.Fa 


\section{INTRODUCTION}

The study of atom ionization in high-velocity collisions with charged particles, where the projectile (collision) velocity $v_{p}$ is much larger than a typical velocity $v_{0}$ of atomic (active) electrons in the target, has a long history (see e.g. [1], [2], [3] and references therein). Starting with a pioneering work of Bethe [4] the first Born approximation has been widely used to describe atom single ionization in such collisions. Within this approximation the initial and final states of the colliding system are approximated by corresponding unperturbed projectile and target wavefunctions and the collision occurs due to just a "single interaction" (or single-virtual-photon exchange) between the projectile and target.

Subsequently it was recognized that more sophisticated approaches, e.g. distorted wave models, close-coupling calculations, eikonal and sudden approximations, with a better treatment of the projectile-target interaction have to be used to consider ionization by highly charged ions (see e.g. [2], [3], [5] and references therein), where even for collision velocities approaching the speed of light the ratio $Z_{p} / v_{p}$ may not be small because of very large values of the projectile charge $Z_{p}$. However, for high-velocity collisions with low-charged projectiles when $\left|Z_{p}\right| / v_{p} \ll 1$ it is commonly anticipated that already the first order approximation leads to a fairly good understanding of that part of single ionization process which is accompanied by emission of relatively slow electrons contributing most to the total cross section ${ }^{1}$, except for collisions where the projectile scatters to angles larger than $m_{e} / M_{p}\left(m_{e}\right.$ and $M_{p}$ are the electron and projectile masses, respectively) and where the projectile interaction with the target nucleus plays a crucial role [6].

In the present paper we consider hydrogen and helium single ionization by bare ions in high-velocity (but still nonrelativistic) collisions in the perturbative regime which we define, somewhat arbitrarily, as $\left|Z_{p}\right| / v_{p} \lesssim 0.1$. We shall restrict our attention to emission of so called soft electrons, which contribute most to the total emission and have typical energies of the order of or smaller than the electron binding energy in the initial target state, and consider only collisions in which the absolute change in the projectile momentum does not exceed a few atomic units, i.e. collisions in which the emission of soft electrons is most likely. Our main goal is to attempt to find out when the first Born approximation can fail in describing essential details of such collisions in the perturbative regime. The fact that such a failure can be the case for emission in the plane, which is perpendicular to the collision plane and contains the incoming projectile momentum, was recently suggested in [7], [8]. Since we are interested in the theoretical study of effects arising from the treatment of the projectile-target interaction beyond the first order, an atomic hydrogen target is very convenient. Hydrogen states are known exactly and the effects of the higher-order terms in the projectile-target interaction are not influenced or even completely masked by the application of improper approximate target states. In addition, since hydrogen contains only one electron the role of the interaction between the projectile and the hydrogen nucleus, which according to the first order approximation should be of no importance, can be explored in detail. Therefore,

\footnotetext{
${ }^{1}$ Note that, in contrast to single ionization, a noticeable difference is observed for the total cross section of double ionization by collisions with protons and antiprotons even at $v_{p} \gtrsim 20-30$ a.u. (see e.g. fig. 7.6 on page 167 of [2] and references to original papers therein).
} 
we will deal mainly with hydrogen ionization.

In addition, helium single ionization in the perturbative regime will also be touched upon using a rather simple choice of helium target states. In this case, however, calculated results for the emission in the plane perpendicular to the momentum transfer are in a sharp disagreement with available experimental data. The analysis of this situation suggests that a proper description of the emission in this plane could be rather demanding with respect to the quality of approximations used for the description of the target states.

The paper is organized as follows. In section II we briefly review the first and second order Born approximations as well as the eikonal (Glauber) and CDW-EIS approaches for collisions with hydrogen. Results and discussion are presented in section III. Section IV contains conclusions.

Atomic units are used throughout except where otherwise stated.

\section{GENERAL CONSIDERATION}

Let us consider a collision between a point-like charged projectile with a charge $Z_{p}$ and a hydrogen atom. Initially the projectile has the momentum $\mathbf{P}_{i}$ and the hydrogen atom is at rest in the ground (internal) state $\psi_{0}$ with an energy $\varepsilon_{0}$. As a result of the collision the projectile changes its momentum to $\mathbf{P}_{f}$ and the target undergoes a transition into a final (internal) state $\psi_{\mathbf{k}}$ which is a continuum target state with an energy $\varepsilon_{k}=k^{2} / 2$ and $\mathbf{k}$ is the final electron momentum with respect to the target nucleus. Note that for collisions with not too large momentum transfers, which are considered in the present paper, cross sections are practically independent of masses of the projectile-ion and the target nucleus.

\section{A. First Born approximation}

The simplest theoretical treatment of the above collision process is given within the standard first Born approximation. According to this approximation the transition amplitude can be written (see e.g. [9], [3]) as

$$
A_{\mathbf{k} 0}^{1 B}=-\frac{Z_{p}}{2 \pi^{2}} \frac{\left\langle\psi_{\mathbf{k}}^{(-)}(\mathbf{r})|\exp (i \mathbf{q} \cdot \mathbf{r})| \psi_{0}(\mathbf{r})\right\rangle}{q^{2}}
$$

Here $\mathbf{r}$ is the electron coordinate with respect to the target nucleus, $\mathbf{q}=\mathbf{P}_{i}-\mathbf{P}_{f}$ is the momentum transfer to the target. According to the first Born approximation the projectile interaction with the target nucleus does not contribute to inelastic transitions (see e.g. [9], [3]) and the momentum transfer to the target is fully due to the single interaction between the projectile and the (initially) bound electron. The momentum transfer is commonly written as $\mathbf{q}=\left(\mathbf{Q}, q_{\text {min }}\right)$ where $\mathbf{Q}$ is the transverse part of the momentum transfer $\left(\mathbf{Q} \perp \mathbf{P}_{i}\right)$ and $q_{\text {min }}$ is the longitudinal momentum transfer component. In collisions with heavy projectiles $q_{\text {min }}=\Delta \varepsilon / v_{p}$, where $\Delta \varepsilon=\varepsilon_{k}-\varepsilon_{0}$ is the energy transfer to the target. Taking into account (1) the fully differential first Born cross section for ionization is given by

$$
\frac{d^{5} \sigma_{1 B}}{d^{3} \mathbf{k} d^{2} \mathbf{Q}}=\frac{4 Z_{p}^{2}}{v_{p}^{2}} \frac{\left|\left\langle\psi_{\mathbf{k}}^{(-)}(\mathbf{r})|\exp (i \mathbf{q} \cdot \mathbf{r})| \psi_{0}(\mathbf{r})\right\rangle\right|^{2}}{q^{4}}
$$




\section{B. Second Born approximation}

Within the approximation which includes the first and second order terms in the projectile-hydrogen interaction the transition amplitude reads

$$
A_{\mathbf{k} 0}^{(1+2) B}=A_{\mathbf{k} 0}^{1 B}+A_{\mathbf{k} 0}^{2 B},
$$

where $A_{\mathbf{k} 0}^{1 B}$ is given by Eq.(1) and the second order transition amplitude is defined as

$$
A_{\mathbf{k} 0}^{2 B}=\left(\frac{Z_{p}}{2 \pi^{2}}\right)^{2} \sum_{n} \int d^{3} \mathbf{P} \frac{\left\langle\psi_{\mathbf{k}}\left|1-\exp \left(i\left(\mathbf{P}_{i}-\mathbf{P}\right) \cdot \mathbf{r}\right)\right| \psi_{n}\right\rangle\left\langle\psi_{n}\left|1-\exp \left(i\left(\mathbf{P}-\mathbf{P}_{f}\right) \cdot \mathbf{r}\right)\right| \psi_{0}\right\rangle}{\left|\mathbf{P}_{i}-\mathbf{P}\right|^{2}\left|\mathbf{P}-\mathbf{P}_{f}\right|^{2}\left(E_{i}+\varepsilon_{0}-E-\varepsilon_{n}+i 0\right)} .
$$

The integration in (4) runs over the intermediate momentum $\mathbf{P}$ of the projectile, $E_{i}$ and $E$ are the projectile energies in the initial and intermediate states, respectively. Further, the sum in (4) is to be taken over all intermediate target states including continuum ones, $\varepsilon_{n}$ is the target energy in an intermediate state $\psi_{n}$.

With the help of Eqs.(1) and (3)-(4) the corresponding cross section is written as

$$
\frac{d^{5} \sigma_{2 B}}{d^{3} \mathbf{k} d^{2} \mathbf{Q}}=\frac{d^{5} \sigma_{1 B}}{d^{3} \mathbf{k} d^{2} \mathbf{Q}}\left(1+2 \operatorname{Re}\left(A_{\mathbf{k} 0}^{2 B} / A_{\mathbf{k} 0}^{1 B}\right)\right),
$$

where the first order cross section $\frac{d^{5} \sigma_{1 B}}{d^{3} \mathbf{k} d^{2} \mathbf{Q}}$ is defined by Eq.(2). The cross section (5) contains terms proportional to $Z_{p}^{2}$ and $Z_{p}^{3}$ and, thus, suggests that, in contrast to the prediction of the first Born approximation, the collision process is not symmetric with respect to the replacement $Z_{p} \rightarrow-Z_{p}$, i.e. that collisions with particles and antiparticles lead to different results. At this point we also note that in general one is not allowed to keep in the cross section (5) terms proportional to $Z_{p}^{4}$ which would arise from $\left(\operatorname{Re}\left(A_{\mathbf{k} 0}^{2 B} / A_{\mathbf{k} 0}^{1 B}\right)\right)^{2}$ and $\left(\operatorname{Im}\left(A_{\mathbf{k} 0}^{2 B} / A_{\mathbf{k} 0}^{1 B}\right)\right)^{2}$. This is because in a more refined treatment, which includes the consideration of the first-, second- and third-order contributions to the transition amplitude, terms in the cross section proportional to $Z_{p}^{4}$ would appear not only from $\left|A_{\mathbf{k} 0}^{2 B}\right|^{2}$ but also due to the product of the first- and third-order Born amplitudes but the latter amplitude is not considered here.

The difficulty in the calculation of the second order transition amplitude (4) arises from the necessity to deal with the infinite sum (and integral) over the intermediate target states. Such a difficulty is removed in a simplified version of the second Born approximation which will be used below. In this version the closure approximation is applied to perform the summation over the intermediate states. Namely, target energies in the intermediate states are replaced by a mean excitation energy $\overline{\varepsilon_{n}}$ and then the sum is evaluated by using the completeness of the target states. By applying the closure approximation the transition amplitude (4) is strongly simplified and is given by

$$
A_{\mathbf{k} 0}^{2 B}=\left(\frac{Z_{p}}{2 \pi^{2}}\right)^{2} \int d^{3} \mathbf{P} \frac{\left\langle\psi_{\mathbf{k}}\left|1-\exp \left(i\left(\mathbf{P}_{i}-\mathbf{P}\right) \cdot \mathbf{r}\right)-\exp \left(i\left(\mathbf{P}-\mathbf{P}_{f}\right) \cdot \mathbf{r}\right)+\exp (i \mathbf{q} \cdot \mathbf{r})\right| \psi_{0}\right\rangle}{\left|\mathbf{P}_{i}-\mathbf{P}\right|^{2}\left|\mathbf{P}-\mathbf{P}_{f}\right|^{2}\left(E_{i}+\varepsilon_{0}-E-\overline{\varepsilon_{n}}+i 0\right)}
$$

The terms in (6) (as well as the corresponding terms in (4)) have simple physical meaning. The parts of the transition matrix element containing $\exp \left(i\left(\mathbf{P}-\mathbf{P}_{f}\right) \cdot \mathbf{r}\right)$ and $\exp \left(i\left(\mathbf{P}_{i}-\mathbf{P}\right) \cdot \mathbf{r}\right)$ 
describe collisions in which the projectile interacts with the target electron and nucleus ( $p$ $e-p$ - $n$ interactions), where each interaction is due to the single (virtual) photon exchange. The term with $\exp (i \mathbf{q} \cdot \mathbf{r})$ appears due to the two interactions between the projectile and the target electron $(p-e-p$-e interactions). The part of the transition matrix element which contains 1 describes the contribution to the process from the two interactions between the projectile and the target nucleus. For inelastic collisions the latter contribution vanishes.

Within the simplified version of the second Born approximation, which employs the closure approximation, the mean excitation energy $\overline{\varepsilon_{n}}$ represents a free parameter. In the present second Born calculations we have considered two options: i) $\overline{\varepsilon_{n}}=0$ and ii) $\overline{\varepsilon_{n}}=$ $0.5\left(\varepsilon_{0}+\varepsilon_{\mathbf{k}}\right)$. The first option has been considered because it was shown to work well for ionization by electrons in asymmetric geometries when initial and final energies of the projectile-electron are much larger than that of the emitted electron and the momentum transfer is relatively small [10]. The second option, in all cases tested, yielded results which differed by not more than few per cent from those obtained with the first one. The choice $\overline{\varepsilon_{n}}=0.5\left(\varepsilon_{0}+\varepsilon_{\mathbf{k}}\right)$, however, allowed in general to reach faster convergence for our numerical results. Second Born results, reported in section III, have been obtained using the option ii).

In general, it is difficult to estimate the accuracy of the simplified second Born approximation. Some ideas about this point could be obtained by comparing its results with those given by experiment and/or obtained by using different approximations. We shall return to this point later (in subsection A of Section III).

\section{Glauber approximation}

In high-velocity collisions eikonal-like approaches are often very useful. One of the most frequently applied eikonal approaches is the Glauber approximation [11]. This approximation fully accounts for the first Born term and, in an approximate manner, takes into account also contributions from all multiphoton exchanges between the projectile and the target. In the Glauber approximation the fully differential cross section for the projectile-hydrogen collisions reads

$$
\frac{d^{5} \sigma_{G l}}{d^{3} \mathbf{k} d^{2} \mathbf{Q}}=\frac{|T(\mathbf{q}, \mathbf{k})|^{2}}{4 \pi^{2}}
$$

where

$$
T(\mathbf{q}, \mathbf{k})=\int d^{2} \mathbf{b} b^{2 i \eta_{p}} \exp (i \mathbf{q} \cdot \mathbf{b})\left\langle\psi_{\mathbf{k}}^{(-)}|| \mathbf{b}-\left.\boldsymbol{\rho}\right|^{-2 i \eta_{p}} \mid \psi_{0}\right\rangle .
$$

In Eq. (8) $\eta_{p}=Z_{p} / v_{p}$, b is the "impact parameter" of the projectile, $\boldsymbol{\rho}$ is the two-dimensional part of the electron coordinate $\mathbf{r}$ which lies in the plane $(\mathbf{q}, \mathbf{b})$ (in order to obtain (8) the eikonal path was chosen to be perpendicular to the total momentum transfer $\mathbf{q}^{2}$ ).

\footnotetext{
${ }^{2}$ We note that, in order to keep $q_{\min }$ nonzero, this path should not be taken as parallel to the initial projectile momentum $\mathbf{P}_{i}$. For the discussion of the choice of the integration path see e.g. reviews [21].
} 
It is well known that, by keeping only the first term in the expansion over $\eta_{p}$ in the Glauber transition amplitude, the latter is reduced to the first Born one. In addition, the Glauber cross section (7)-(8) has an interesting and useful property. It is easily shown that if one neglects the interaction between the projectile and the target nucleus then the Glauber cross section for collisions with single-electron targets becomes equivalent to the first Born cross section despite the former attempts to account for all multiphoton exchanges between the projectile and the target electron and the latter considers only the singlephoton exchange. The origin of this property is a well known peculiarity of the Coulomb interaction: both first order and exact treatments yield identical cross sections for collisions of two charged particles. The Glauber approximation being generally superior to the first order approach also yields the same cross sections in this case [11]. Of course, this property does not mean that according to the Glauber approximation the projectile interaction with the electron in hydrogen is always well described by just single photon exchange and that all non-first-order effects in hydrogen ionization are contained only in the interaction between the projectile and the hydrogen nucleus. Yet, this property can be used to get some ideas about the role of the latter interaction for electron emission in high-velocity collisions.

\section{CDW-EIS approximation}

The CDW-EIS approximation was introduced in [12] by replacing the CDW description of the initial state in the CDW-CDW model [13] by its asymptotic (eikonal) form. This approximation belongs to the family of perturbative distorted-wave theories and is rather well documented in the literature (see [12], [14], [15] and references therein, and also [19]).

Within the CDW-EIS approximation the transition amplitude for ionization by a heavy projectile is written as [15]

$$
T^{C D W-E I S}=-\left\langle\varphi \mathbf{P}_{f} \psi_{\mathbf{k}} \chi_{f}^{C D W}\left|\nabla_{\mathbf{r}_{T}} \cdot \nabla_{\mathbf{r}_{P}}\right| \varphi_{\mathbf{P}_{i}} \psi_{0} \chi_{i}^{E I S}\right\rangle
$$

In the above expression $\varphi_{\mathbf{P}_{i, f}}$ are plane waves describing the free relative internuclear motion in the initial and final states, $\psi_{0}$ and $\psi_{\mathbf{k}}$ are the initial and final (free) target states. Within the CDW-EIS approach the operator causing transitions is the so called nonorthogonal kinetic energy $\boldsymbol{\nabla}_{\mathbf{r}_{T}} \cdot \boldsymbol{\nabla}_{\mathbf{r}_{P}}$, where $\mathbf{r}_{T}$ and $\mathbf{r}_{P}$ are the electron position with respect to the target nucleus and projectile, respectively.

The final state of the colliding particles includes the distortion through the function

$$
\begin{aligned}
\chi_{f}^{C D W}= & \exp \left(-\pi Z_{p} Z_{t} /(2 P)\right) \Gamma\left(1-i Z_{p} Z_{t} / v_{p}\right) F_{1}\left(i Z_{p} Z_{t} / v_{p} ; 1 ;-i P R-i \mathbf{P} \cdot \mathbf{R}\right) \\
& \exp \left(\pi Z_{p} /(2 \kappa)\right) \Gamma\left(1+i Z_{p} / \kappa\right) F_{1}\left(-i Z_{p} / v_{p} ; 1 ;-i \kappa r_{P}-i \boldsymbol{\kappa} \cdot \mathbf{r}_{P}\right) .
\end{aligned}
$$

Here $\mathbf{P}$ is the relative internuclear linear momentum, $\mathbf{R}$ the internuclear separation and $\boldsymbol{\kappa}=\mathbf{k}-\mathbf{v}_{p}$ is the momentum of the emitted electron with respect to the projectile. Further, in (10) $\Gamma$ is the gamma-function and $F_{1}$ is the confluent hypergeometric function.

The eikonal factor in the initial state reads

$$
\chi_{i}^{E I S}=\exp \left(i \frac{Z_{p} Z_{t}}{v_{p}} \log \left(v_{p} R-\mathbf{v}_{p} \cdot \mathbf{R}\right)\right) \exp \left(-i \frac{Z_{p}}{v_{p}} \log \left(v_{p} r_{P}-\mathbf{v}_{p} \cdot \mathbf{r}_{P}\right)\right) .
$$


Most often the CDW-EIS approach is used in such a form (semiclassical form), in which the projectile interaction with the target nucleus (target core) is neglected ( [12], [14], [3], and references therein). For heavy ion-atom collisions this form has been very successful in describing total ionization cross sections and electron emission spectra. The "full" version of the CDW-EIS approach, where the projectile-target nucleus interaction is included, has yielded quite good results for cross sections of helium ionization by protons differential in the projectile scattering angle [16].

In the present paper we shall apply the "full" version of the CDW-EIS approximation which including the nuclear-nuclear interaction into account. The latter interaction shall be dealt with within the eikonal approximation. In such an approximation the distortion due to the $n-n$ interaction is accounted for by an eikonal factor, representing the asymptotics of the corresponding two-body Coulomb wave, not only in the initial but also in the final channel [16] (see also [12]). Such an approximation is quite reasonable as long as the projectile deflection is very small and, simultaneously, the velocity of the recoil ion remains negligible compared to that of the emitted electron. In the case under consideration such conditions are, of course, fulfilled for a vast majority of ionizing collisions.

\section{RESULTS AND DISCUSSION}

\section{A. Hydrogen ionization}

It shall be seen below that the Glauber, the CDW-EIS and (the simplified version of) the second order approximations predict that in the perturbative regime some considerable deviations from the first order results are possible. The important question to address, therefore, is how reliable are such predictions, i.e. whether these approximations work well enough for ionization by fast ions in the perturbative regime.

Both the Glauber and second order approximations were studied in great detail for potential and elastic scattering and also for inelastic collisions where the target undergoes a transition into some of its first excited bound states, the advantages and shortcomings of these approximation in such cases are known (see [21], [22] and references therein). In case of ionization, to our knowledge, the situation, in general, is less clear.

It was found that the second Born approximation (with the closure) yields rather good results for hydrogen and helium ionization by fast electron impact in asymmetric collision geometries where the momentum transfer is small and the emitted electron is a soft electron [10], [22]. In the present paper we also consider soft electron emission in collisions with relatively small momentum transfers and, therefore, one can expect that in our case the second Born approximation should yield reliable results.

The main deficiency of the Glauber approximation in the case of weak perturbations $Z_{p} / v_{p} \ll 1$ is that the second term of the expansion of the Glauber transition amplitude, which is proportional to $Z_{p}^{2}$, in general does not fully reproduce its second Born counterpart. However, in the present study of collisions with fast ions, where the momentum transfer as well as the energy of emitted electron are relatively small, it turned out that results obtained using (5)-(6) and (7)-(8) are quite similar in the perturbative regime. Moreover, this similarity remains if one uses the cross section 


$$
\frac{d^{5} \sigma_{2 B}}{d^{3} \mathbf{k} d^{2} \mathbf{Q}}=\frac{d^{5} \sigma_{1 B}}{d^{3} \mathbf{k} d^{2} \mathbf{Q}}\left|1+A_{\mathbf{k} 0}^{2 B} / A_{\mathbf{k} 0}^{1 B}\right|^{2}
$$

instead of (5). The cross section (12) was taken for calculating hydrogen and helium ionization by an electron impact by the authors of [10] which found that for asymmetric collision geometries, where the momentum transfer to the target is small and the energy of emitted electron is much less than that of the incident electron, the third Born contribution to the transition amplitude is of minor importance for the emission cross section. In our case, where one deals with (highly) asymmetric geometries but for collisions with ions, we did not consider the third Born contribution to the transition amplitude. We, however, performed calculations using Eqs.(5) and (12) and did not find any considerable differences between results obtained with $(5)$ and (12). In cases tested both $\left(\operatorname{Re}\left(A_{\mathbf{k} 0}^{2 B} / A_{\mathbf{k} 0}^{1 B}\right)\right)^{2}$ and $\left(\operatorname{Im}\left(A_{\mathbf{k} 0}^{2 B} / A_{\mathbf{k} 0}^{1 B}\right)\right)^{2}$ turned out to be only small corrections to $1+2 \operatorname{Re}\left(A_{\mathbf{k} 0}^{2 B} / A_{\mathbf{k} 0}^{1 B}\right)$. In particular, we observed that both $p-e-p-n$ and $p-e-p$-e interactions give small contributions to the term $\left(\operatorname{Re}\left(A_{\mathbf{k} 0}^{2 B} / A_{\mathbf{k} 0}^{1 B}\right)\right)^{2}$. In contrast, the contributions from the $p-e-p-n$ and $p-e-p-e$ interactions to the term $\left(\operatorname{Im}\left(A_{\mathbf{k} 0}^{2 B} / A_{\mathbf{k} 0}^{1 B}\right)\right)^{2}$ are rather large but in this term they nearly cancel each other.

Such very small contributions of the terms, proportional to $Z_{P}^{4}$, into the cross section (12) and especially the above mentioned nearly cancellation between the $p-e-p$-n and $p-e-p$ - $e$ interactions in $\left(\operatorname{Im}\left(A_{\mathbf{k} 0}^{2 B} / A_{\mathbf{k} 0}^{1 B}\right)\right)^{2}$ may give rise some doubts about whether in our case the cross section (12) accurately accounts for the $Z_{P}^{4}$ term since the additional $Z_{p}^{4}$ contribution would appear due to the product of the first and third Born transition amplitudes. Therefore, our discussion of the second Born results will be based on the cross section (5) which is accurate up to $Z_{p}^{3}$.

The CDW-EIS was "invented" specially for considering ionizing collisions. For such collisions the CDW-EIS is regarded as one of the best theories and, in particular, as being superior to the Glauber approximation. The CDW-EIS was very successfully applied to calculate total ionization cross sections and spectra of emitted electrons even in collisions with highly charged ions where the effective perturbation strength $Z_{p} / v_{p}$ was substantially larger than 1. Further, the "full" version of the CDW-EIS, which includes the projectile interaction with the target nucleus, was used in [17], [18] to calculate the fully differential cross section for hydrogen ionization by electron impact in cases where $\left|Z_{p}\right| / v_{p}$ was not much smaller than 1. A good agrement with experimental data was reported.

Thus, summarizing the above brief discussion, one can note the following. Of course, we do not exactly know the accuracy of the second order, Glauber and CDW-EIS approaches in our case. However, we would like to point out that: i) the CDW-EIS method seems to be well suited for the present study; ii) the second Born approximation (with using the closure approximation) is known to yield good description for ionization by electrons in asymmetric geometries; iii) in cases of weak perturbations, $\left|Z_{p}\right| / v_{p} \ll 1$, it turned out that the second Born and CDW-EIS approximations yield quite similar results; iv) as shall be seen below in all cases studied in the present paper the CDW-EIS approach and Glauber approximation give very close (sometimes even undistinguishable) results. Since all these three approximations are quite different, the application of them to the same problem could be thought 
of as representing some (indirect) cross-checks for each other. Therefore, there seem to be grounds to believe that results of these approximations in the case under consideration are reliable enough to make conclusions about the role of the non-first-order effects in the electron emission by collisions with fast ions.

\section{Fully resolved cross section}

\section{Emission in the collision plane.}

The first Born approximation is known to yield good results for the total cross section for single ionization provided $\left|Z_{p}\right| / v_{p} \ll 1$. The main part of the electron emission occurs in the collision plane containing the incoming projectile momentum, which is taken along the $z$-axis (see figure 1), and the momentum transfer. Therefore, it is usually stated that the first Born approximation provides a good description of collision physics in this plane [7], [23]. It turns out, however, that this is not always the case. Let us consider, as an example, collisions with $100 \mathrm{MeV} / \mathrm{u} \mathrm{C}^{6+3}$ and equivelocity anticarbon nuclei $\bar{C}^{6-}\left(v_{p} \approx 60,\left|Z_{p}\right| / v_{p} \approx 0.1\right)$. Results of our calculations for the fully differential cross section, $d^{5} \sigma /\left(k^{2} d k d \Omega_{k} Q d Q d \varphi_{Q}\right)$, for emission of a low-energy electron in the collision plane $\left(\varphi_{k}=0^{0}\right)$ are displayed in figure 2. For this case the first Born approximation predicts that the ratio of the binary peak maximum to the recoil peak maximum is close to 5 and is, of course, independent of the charge of the projectile. However, calculations using the Glauber, CDW-EIS and second Born approximations, respectively, yielding rather similar results suggest that this ratio should be close to 6.3 in collisions with $\mathrm{C}^{6+}$ and to 3.8 for collisions with $\bar{C}^{6-}$. Thus, all these three calculations predict a rather strong dependence on the sign of the projectile charge which is a clear signature of the higher-order contributions.

We noted already that the Glauber approximation gives exactly the first order cross section provided the interaction between the projectile and the hydrogen nucleus is neglected. Within the CDW-EIS approach the neglect of the latter interaction, generally speaking, does not lead to the first order cross section. However, in the case under consideration, where the effective perturbation strength $\left|Z_{p}\right| / v_{p}$ is very small, the neglect of the nuclear-nuclear interaction in the CDW-EIS calculation resulted in the emission pattern very close to that given by the first order calculation. In addition, the analysis of the importance of the term in the second order transition amplitude (6), which describes the two interactions between the projectile and the target electron, shows that this term influences rather weakly the cross section (5). Therefore, one can conclude that it is the projectile interaction with the hydrogen nucleus, occurring via single-photon exchange in the case under consideration, which is mainly responsible for the deviations from the first order results in the collision plane. Previously, clear signatures of the projectile interaction with the target nucleus in target single ionization by fast ions were reported only for relatively large projectile scattering

\footnotetext{
${ }^{3}$ Such projectiles were recently used in [7], [8] and [23] to study the various aspects of helium single ionization in the perturbative limit in collisions with heavy particles.
} 
angles [6] corresponding to much higher transverse momentum transfers $\left(Q \gtrsim v_{p}\right)$ compared to those considered in the present paper.

We have discussed an example of electron emission in the plane $\varphi_{k}=0^{0}$ in collisions with carbon and anticarbon nuclei, the latter being quite exotic. However, similar conclusions about the role of the interaction between the projectile and the hydrogen nucleus for the emission pattern in the collision plane follow also for collisions with protons and antiprotons at similar values of $\left|Z_{p}\right| / v_{p}$ (see figure 3 ) since the cross section scales with $Z_{p} / v_{p}$.

\section{Emission in the plane $\varphi_{k}=90^{\circ}$ (perpendicular plane).}

According to the first Born approximation electron emission in the plane perpendicular to the momentum transfer $\mathbf{q}$ is strongly suppressed compared to emission in the collision plane. Therefore, one could expect that the emission pattern in this plane may be even more sensitive to the higher-order contributions from the projectile-target interaction compared to the case with emission in the collision plane [7], [8]. In high-velocity collisions the minimum momentum transfer $q_{\min }=\left(k^{2}+1\right) /\left(2 v_{p}\right)$ is quite small for the majority of the emitted soft electrons which have momenta $k \lesssim 1$ a.u.. Therefore, for transverse momentum transfers $Q$ which are not much smaller than 1 a.u. one has $\mathbf{q} \approx \mathbf{Q}$ and a plane defined by $\varphi_{k}=90^{\circ}$ (the angle $\varphi_{\mathbf{k}}=0^{0}$ corresponds to electron emission along $\mathbf{Q}$, see figure 1) practically represents the perpendicular plane. According to the first order results the emission pattern should be a constant quantity in the plane perpendicular to q (see e.g. figure 4). In figure 4 we display our results for the fully differential cross section in collisions with the same projectiles as before $\left(100 \mathrm{MeV} / \mathrm{u} \mathrm{C}^{6+}\right.$ and $\left.\bar{C}^{6-}\right)$ but now for the electron emission angle $\varphi_{\mathbf{k}}=90^{0}$ and energy $\varepsilon_{k}=10 \mathrm{eV}$. Whereas the first order calculation gives (nearly) a constant value for the emission cross section, the second order, Glauber and CDW-EIS approximations suggest that the cross section is rather far from being a constant. All these three approximations yield quite close results and predict that for collisions with $\mathrm{C}^{6+}$ projectiles the emission pattern shows two pronounced minima centered at $\vartheta_{\mathbf{k}}=90^{\circ}$ and $270^{\circ}$ whereas for collisions with equivelocity $\bar{C}^{6-}$ projectiles one observes maximum of emission at these angles. Thus, the emission pattern in the perpendicular plane displays a strong charge-asymmetry effect.

It can be shown analytically that in the simplified version of the second Born approximation, which is used here, the term in the transition amplitude (6), corresponding to the two interactions between the projectile and the target electron, depends only on the absolute values of the momentum transfer $\mathbf{q}$ and the electron momentum $\mathbf{k}$. Further, if one neglects the $n-n$ interaction in the CDW-EIS calculation then the emission pattern in the perpendicular plane turns out to be very similar to that given by the first Born calculation. In addition, as was already noted, within the Glauber approximation the omission of the $n-n$ interaction leads to first Born results. Therefore, according to all non-first-order calculations of the present paper, in the perturbative regime $\left|Z_{p}\right| / v_{p} \ll 1$ the origin of the nonconstant behaviour of the cross section in the perpendicular plane as well as of the charge asymmetry effect lies in the interaction (via single-photon exchange) between the projectile and the target nucleus.

It worth while to mention that, according to our analysis of relativistic collisions with hydrogen at $Q \sim 1 \gg q_{m i n}$, the emission pattern in the plane $\varphi_{k}=90^{\circ}$ is not a constant even for collisions with protons and antiprotons (or electrons and positrons) moving at 
velocities very close to the speed of light (see figure 5) and still shows a noticeable effect of the projectile charge sign.

It is interesting to note that when the effective strength of the perturbation, $\left|Z_{p}\right| / v_{p}$, increases the emission pattern in the perpendicular plane, according to both the Glauber and CDW-EIS approximations, should change drastically for collisions with projectiles having $Z_{p}>0$ (see figure 6). Now, instead of minimum, one observes a maximum at the angle $\vartheta_{k}=90^{\circ}$ (and $\vartheta_{k}=270^{\circ}$ ) for collisions with positively charged projectiles. For collisions with antiprojectiles the maximum would remain becoming higher and more narrow. For a collision velocity of 60 a.u. considered in figure 6 the second order approach (results of which are not shown in the figure) begins to definitely fail already at $Z_{p}=15$ leading to negative values for the cross section (5). This suggests that starting with $Z_{p} / v_{p} \approx 0.2-0.25$ multiple photon exchanges with both the target electron and nucleus start to play a very important role in forming the emission pattern. The convergence of the Born series is getting worse with increasing $\left|Z_{p}\right| / v_{p}$ and perhaps becomes rather questionable for $\left|Z_{p}\right| / v_{p}=0.5$. On the other hand, the CDW-EIS approximation should be valid in this case. In addition, the Glauber approximation is also expected to work quite well in the case considered in figure 6 where the collision velocity is very high, the perturbation is relatively large and the momentum transfer $q \approx 1 \mathrm{a}$.u. is nearly perpendicular to the initial projectile momentum (about the accuracy of the Glauber approximation in the case of relatively strong perturbations see also the reviews by Joachain and Quigg and Byron and Joachain in [21]).

\section{On the role of the higher-order effects in the perturbative regime.}

By analyzing collisions with different momentum transfers $Q$ and electron emission energies we found the following main trends for the role of higher-order effects in the fully resolved electron emission pattern in the perturbative regime (for this regime it is in essence the role of the interaction between the projectile and the hydrogen nucleus via single-photon exchange). First, on average, their importance increases with increasing the transverse part of the momentum transfer at a fixed emission energy. Second, in collisions with a fixed momentum transfer $Q \sim 1$ the deviations from first order results in the collision plane increase when the electron emission energy $\varepsilon_{k}$ decreases. These deviations become especially obvious when the electron momentum in the final state $k$ is substantially smaller than the momentum transfer $q \approx Q$. Third, in collisions with fixed momentum transfer $Q$ and emission energy $\varepsilon_{k}$ the deviations from first order results increase when the angle $\varphi_{k}$ increases from $0^{0}$ to $90^{\circ}$. Fourth, the role of higher-order effects is, on average, noticeably larger for collisions with negatively charged projectiles.

The first point looks almost trivial since collisions with larger $Q$ generally correspond to smaller impact parameters where the projectile-target interaction is expected to be stronger. The second point tells us that in collisions, where the main part of the momentum transfer (finally) goes to the recoil ion, the "direct interaction" between the projectile and the target nucleus is necessary since the electron in such a case may not always be able to act as an effective mediator in transferring a relatively large recoil momentum to the target nucleus. The third point is also not very unexpected since usually if the first order transition amplitude becomes suppressed because of some factors then the higher-order transition amplitudes begin to play a more important role. The first three points can, to some extent, 
be summarized in a statement that the role of the interaction between the projectile and the target nucleus in the perturbative regime becomes important in such collisions which are characterized by momentum transfers of the order of or higher than a typical electron momentum in the target ground state and, simultaneously, are quite far from the so called Bethe ridge describing binary (projectile-electron) encounters.

The fourth point does not seem to be easy to understand since intuitively one expects that the trajectories of very fast and heavy projectiles are very similar for both $Z_{p}>0$ and $Z_{p}<0$. At this point one can note that a similar situation, namely stronger higher-order effects in collisions with negatively charged projectiles, holds for double ionization where the double-to-single ionization cross section ratio converges much faster to the so-called highvelocity limit, which is described by first order theories, in collisions with protons compared to those with antiprotons (see e.g. [2] and references therein).

\section{Integrated cross sections}

The pronounced difference between the first order results and those obtained by using the second order, Glauber and CDW-EIS approximations, which was found for the fully differential cross section, rapidly decreases when one starts to integrate the cross section. That this will be the case is seen already from figure 2 which shows that after the summing over all emission angles $\vartheta_{k}$ the difference seems to substantially decrease ${ }^{4}$. This is confirmed by calculations which, in particular, show that the difference between the first and nonfirst order results in the perturbative regime practically completely disappears after the integration over the electron emission angles. As an example, we show in figure 7 the cross section differential in the electron energy and (the absolute value of ) the transverse momentum transfer $Q$. This cross section is given as a function of $Q$ for a fixed electron emission energy of $10 \mathrm{eV}$. It is seen in the figure that only at $Q \gtrsim 2.5 \mathrm{a}$.u. there starts to appear a considerable difference between the first order results and those given by the Glauber and CDW-EIS approaches ${ }^{5}$. The difference becomes quite substantial at $Q \gtrsim 3$ a.u.. However, the region of these, relatively large, momentum transfers is only of minor importance for the total emission. Thus, the non-first order effects in the total cross section as well as in energy and momentum spectra of the emitted electron turn out to be quite small. This is in accord with expectations that in the perturbative regime the first order approaches are quite valid to calculate spectra of soft electrons and the total electron emission for the process of target single ionization.

\footnotetext{
${ }^{4}$ In this respect the situation is different for emission in the plane $\varphi_{k}=90^{0}$ where the difference with first order result does not seem to become much smaller after summing over $\vartheta_{k}$. This plane, however, contributes little to the total emission.
}

${ }^{5}$ Note that the latter two yield practically identical results. 


\section{B. Helium single ionization}

There exist experimental data on fully resolved cross sections for helium single ionization by $100 \mathrm{C}^{6+}$ [7], [8], [23]. Therefore, we have attempted to analyze single ionization of helium in such collisions by using the first order and Glauber approximations in order to account for the projectile-helium interaction.

In case of helium ionization the projectile-target interaction involves four particles. Already because of this factor the analysis of the origin of higher-order effects, i.e. is it an additional interaction with the target nucleus or with the "passive" target electron etc, would become more complicated. In addition, one faces the problem of an appropriate description of the initial and final helium states. In our present study we used the following two-electron approximate states

$$
\psi_{0}\left(\mathbf{r}_{1}, \mathbf{r}_{2}\right)=N_{0}\left(\exp \left(-\alpha r_{1}-\beta r_{2}\right)+\exp \left(-\alpha r_{2}-\beta r_{1}\right)\right)
$$

and

$$
\psi_{\mathbf{k}}\left(\mathbf{r}_{1}, \mathbf{r}_{2}\right)=\xi_{\mathbf{k}}\left(\mathbf{r}_{1}, \mathbf{r}_{2}\right)-\left\langle\psi_{0}\left(\mathbf{r}_{1}, \mathbf{r}_{2}\right) \mid \xi_{\mathbf{k}}\left(\mathbf{r}_{1}, \mathbf{r}_{2}\right)\right\rangle \psi_{0}\left(\mathbf{r}_{1}, \mathbf{r}_{2}\right)
$$

where

$$
\xi_{\mathbf{k}}\left(\mathbf{r}_{1}, \mathbf{r}_{2}\right)=N_{k}\left(\exp \left(-\lambda r_{1}\right) \phi_{\mathbf{k}}\left(\mathbf{r}_{2}, Z_{0}\right)+\exp \left(-\lambda r_{2}\right) \phi_{\mathbf{k}}\left(\mathbf{r}_{1}, Z_{0}\right)\right),
$$

to simulate the initial ground and final continuum helium state, respectively. In (13) the screening parameters are taken as $\alpha=1.18853$ and $\beta=2.18317$. In (15) the choice $\lambda=2$ assumes that the "passive" electron is finally in the ground state of $\mathrm{He}^{+}$and "sees" the unscreened nuclear charge, $\phi_{\mathbf{k}}\left(\mathbf{r}, Z_{0}\right)$ is the Coulomb continuum state which describes the motion of the ionized electron in the field of the residual target ion with an effective charge $Z_{0}$. In (13) and (15) $N_{0}$ and $N_{k}$ are the corresponding normalization factors. The initial and final states (13) and (14) are orthogonal.

The choice (13) and (14) for the helium states is rather simple. Yet, the application of (13) and (14) with the above mentioned values of the parameters $\alpha, \beta$ and $\lambda$ and also with $Z_{0}=1$ allowed to describe quite successfully experimental data on electron spectra emitted in the process of helium single ionization in collisions with $1 \mathrm{GeV} / \mathrm{u} \mathrm{U} \mathrm{U}^{92}$ ions [24].

In the present study of helium ionization by $100 \mathrm{MeV} \mathrm{C}^{6+}$ the use of the states (13) and (14) with $\lambda=2$ and $Z_{0}=1$ allows to quite well reproduce measured electron spectra integrated over the momentum transfer. In addition, the fully resolved emission pattern in the collision plane is reasonably well described by the application of (13) and (14). In this plane we observe differences between results of the first Born and Glauber approximations which are very similar to those found for collisions with hydrogen ${ }^{6}$. These results will not be discussed here.

\footnotetext{
${ }^{6}$ Note that the experimentally studied cases, reported by [23], dealt with close values of the electron and transfer momenta $k \sim Q$ where, according to our calculations, the contributions from higher-order effects is rather small. Because of the experimental uncertainty and since we use approximate helium states one cannot make a decisive conclusion which set of calculations yields better agreement with experiment.
} 
The situation, however, drastically changes if we consider the emission in the plane $\varphi_{k}=90^{\circ}$. According to the eikonal calculations (with $\lambda=2$ and $Z_{0}=1$ ) the emission pattern in collisions with helium should be rather similar to that in collisions with hydrogen, i.e. the calculations predict a minimum for the cross section centered at $\vartheta_{k}=90^{\circ}\left(270^{\circ}\right)$. The experiment, however, yields exactly the opposite, showing that there is a maximum in the emission pattern at $\vartheta_{k}=90^{0}\left(270^{\circ}\right)$ ! The situation becomes even more curious since emission pattern more similar to that observed experimentally appears in the calculations when the projectile charge is negative and, as in the case with hydrogen, a maximum is suggested at $\vartheta_{k}=90^{0}\left(270^{\circ}\right)$.

Any theory, which attempts to describe ion-atom collisions, has to deal with two main points: i) the projectile-target interaction should be properly treated and ii) (initial and final) free target states should be described with reasonable accuracy. Depending on the strength of the projectile-target interaction and on what one is going to describe (the total cross section, electron spectra, the fully differential cross section, etc) the point i) or ii) can be comparatively more (or less) important but, in general, both have to be properly addressed. Moreover, one should note that these two points are not quite independent. Namely, the projectile-target interaction depends on the position of all the particles constituting the target. Therefore, since different approximations for free target states will generally lead to different space distributions of the target electrons, the projectile-target interaction will effectively be dependent on a choice of free target states.

As we have seen in the previous subsection, in high-velocity collisions with hydrogen the Glauber approximation yields results which are practically identical to those given by the CDW-EIS. We believe that the way, in which the projectile-target interaction is treated within the Glauber approximation, is per se accurate enough to be applied for considering helium ionization at high velocities. However, since the above mentioned points i) and ii) are connected, the application of the Glauber approximation for helium ionization should be accompanied by a choice of good wavefunctions for the free helium states. In our opinion, the strict disagreement with experiment for the plane $\varphi_{k}=90^{0}$ could be attributed to the failure of the approximate target states (13) and (14) to describe some rather delicate details in the electron dynamics in the "transition region" where $r_{1} \sim r_{2} \sim 1$ a.u. and which contributes most to the transition matrix element. In particular, the state (15) with $\lambda=2$ and $Z_{0}=1$ is only asymptotically correct, when one electron is bound and the other is far enough from the residual ion. However, in the "transition region" the äctive" electron is still close to the helium nucleus. We performed calculations in which we varied values of the effective charges $\lambda$ and $Z_{0}$ in the final state (15). While by changing $\lambda$ in reasonable limits the emission pattern remains qualitatively the same, we found that when $Z_{0}$ increases and becomes close to 2 the minimum in the calculated cross section at $\vartheta_{k}=90^{\circ}\left(270^{\circ}\right)$ changes to a local maximum.

In the $3 C$ calculations of [8] helium single ionization was considered as an effective three-body problem. Target initial and final states were described in a single electron approximation using Hartree-Fock wavefunctions which for the final state differ substantially from a Coulomb wave with $Z_{0}=1$ in the transition region. Yet, a shallow minimum at $\vartheta_{k}=90^{\circ}\left(270^{\circ}\right)$ rather than a maximum is predicted by the calculations of [8]. We have performed calculations where helium was regarded as a hydrogen-like system with an effective charge of the atomic core $Z_{\text {eff }}$ taken as the same for both initial and final electron 
states. No variation of the value of this charge in reasonable limits could produce even a local maximum at $\vartheta_{k}=90^{\circ}\left(270^{\circ}\right)$.

Summarizing the above brief discussion and the results for hydrogen, reported in subsection A of this section, it is plausible to assume that the experimentally observed structure in the fully differential cross section in the perpendicular plane is a four-body phenomenon and that a proper description of this phenomenon demands rather sophisticated approximations for two-electron initial and final helium states. For example, based on our results for hydrogen ionization in the perturbative regime, one might think that the experimentally observed structure in helium emission could be due to collisions in which the projectile have two interactions with äctive" and "passive" helium electrons and does not directly interact with the helium nucleus. In such a case, since electrons have a negative charge, the results for collisions with positively and negatively charged projectiles would be reversed and one would observe a maximum at $90^{\circ}$ and $270^{\circ}$ in collisions with $Z_{p}>0$.

One should also note that for fast collisions with highly charged ions, where the ratio $Z_{p} / v_{p}$ is not much smaller than 1 , the calculated emission pattern for helium single ionization in the plane $\varphi_{k}=90^{\circ}$ changes similarly to that found for hydrogen ionization. In such a case there appears a pronounced maximum at $\vartheta=90^{\circ}\left(270^{\circ}\right)$ and the emission pattern remains qualitatively the same when using different options to approximate helium states. Thus, in the case of strong perturbations, the emission pattern seems to be not very sensitive to the details of the electron motion in helium.

\section{CONCLUSION}

We have considered hydrogen ionization by charged projectiles in the perturbative regime when $\left|Z_{p}\right| / v_{p} \lesssim 0.1$. To this end we have used the first and second Born, Glauber and CDWEIS approximations. We found that, despite the projectile-target interaction is expected to be quite weak, higher-order contributions from this interaction can still noticeably influence electron emission pattern even in the collision plane, which is often regarded as being very well reproduced by first-order theories. Moreover, these contributions become even more "visible" if one considers electron emission in the plane perpendicular to the momentum transfer. We have attributed the difference between the first and higher-order results mainly to the interaction between the projectile and the hydrogen nucleus. In the perturbative regime the interaction between the projectile and the target nucleus becomes important in such collisions i) where the momentum transfer is not substantially smaller than a typical electron momentum in the target ground state, $p_{0}=1$ a.u., and ii) where, for emission in the collision plane, the main part of the momentum transfer goes to the recoil of the hydrogen nucleus. The higher-order effects in the projectile-target interaction turned out to be on average more pronounced for collisions with negatively charged particles.

The difference with the first order results rapidly decreases when one starts to consider integrated cross sections. In particular, the difference with first order results for electron emission spectra and for the total emission is very small in the perturbative regime.

In case of helium single ionization in the perturbative regime the situation seems to be much more complicated. In particular, our consideration suggests that a proper description of electron emission in the perpendicular plane might be very demanding with respect to the quality of the approximations used to describe the initial and final (free) helium states. 


\section{ACKNOWLEDGEMENT}

The authors acknowledge with thanks the support from the Max-Planck-Gesellshaft and the Deutscheforschungsgemeinshaft (DFG). 


\section{REFERENCES}

[1] N.Bohr, Danske Vidensk. Selsk., Mat.-Phys. Meddr. 18 No 2 (1948)

[2] J.H.McGuire, Electron Correlation Dynamics in Atomic Collisions Cambridge University Press (1997)

[3] N.Stolterfoht, R.D.DuBois, R.D.Rivarola, Electron Emission in Heavy Ion-Atom Collisions Springer Berlin-Heidelberg-New York (1997)

[4] H.Bethe, Ann. Phys. Lpz., 5325 (1930)

[5] P.D.Fainstein, V.H.Ponce and R.D.Rivarola, J.Phys. B 21, 3091 (1991)

[6] E.Y. Kamber, C.L.Cocke, S.Cheng, and S.L.Vargnese, Phys. Rev. Lett. 602026 (1988); J.P.Giese and E.Horsdal, Phys. Rev. A 602018 (1988); R.Dörner, J.Ullrich, O.Jagutzki, S.Lencinas, and H.Schmidt-Böcking, R.Olson, Supplement to Z.Phys. D 2157 (1991); A.Gensmantel, J.Ullrich, R.Dörner, R.E.Olson, K.Ullmann, E.Forberich, S.Lencinas and H.Schmidt-Böcking, Phys.Rev. A 454572 (1992)

[7] M.Schulz et al, J.Phys. B 34 L305 (2001)

[8] M.Schulz, R.Moshammer, D.Fischer, D.H.Madison, S.Jones, and J.Ullrich, Nature 422 48 (2003)

[9] M.R.C.McDowell and J.P.Coleman, Introduction to the theory of Ion-Atom Collisions , North-Holland Publishing Company (Amsterdam), 1970)

[10] F.W.Byron, C.J.Joachain and Piraux J.Phys. B13 L673 (1980), B 15 L293 (1982); H.Ehrhard, M.Fisher, K.Jung, F.W.Byron, C.J.Joachain and B.Piraux, Phys.Rev.Lett. 481807 (1982)

[11] R.J.Glauber, in Lectures in Theoretical Physics p. 315, edited by W.E.Brittin, L.G.Dunham, Interscience Publishers, Inc., New York (1959)

[12] D.S.F.Crothers and J.F.McCann, J.Phys. B 163229 (1983)

[13] Dz.Belkic, J.Phys. B 113529 (1978)

[14] P.D.Fainstein, V.H.Ponce and R.D.Rivarola, J.Phys. B 243091 (1991)

[15] D.S.F.Crothers and L.J.Dube, Adv.At.Mol.Opt.Phys. 30287 (1993)

[16] V.D.Rodrigues, J.Phys. B 29275 (1996)

[17] S.Jones and D.H.Madison, Phys. Rev. Lett. 812886 (1998)

[18] S.Jones and D.H.Madison, Phys. Rev. A 62042701 (2000)

[19] Note that very recently a generalization of the CDW-EIS, GCDW-EIS, was proposed in [20].

[20] D.S.F.Crothers, D.M.McSherry, S.F.C.O'Rourke, M.B.Shah, C.McGrath, and H.B.Gilbody, Phys.Rev.Lett., 88053201 (2002); S.F.C.O'Rourke, D.M.McSherry and D.S.F.Crothers, J.Phys. B 36341 (2003)

[21] E.Gerjuoy and B.K.Thomas, Rep.Progr.Phys., 371345 (1974); C.J.Joachain and C.Quigg, Rev.Mod.Phys. 46279 (1974); F.W.Byron Jr. and C.J.Joachain, Phys.Rep. 34233 (1977); F.T.Chan, M.Lieber, G.Foster and W.Williamson, Adv.Electronics and Electron Physics 49134 (1979)

[22] H.R.J.Walters, Phys.Rep. 1161 (1984)

[23] D.Madison, M.Schulz, S.Jones, M.Foster, R.Moshammer and J.Ullrich, J.Phys. B 35 $3297(2002)$

[24] A.B.Voitkiv, B.Najjari, R.Moshammer and J.Ullrich, A 65032707 (2002) 


\section{FIGURES}

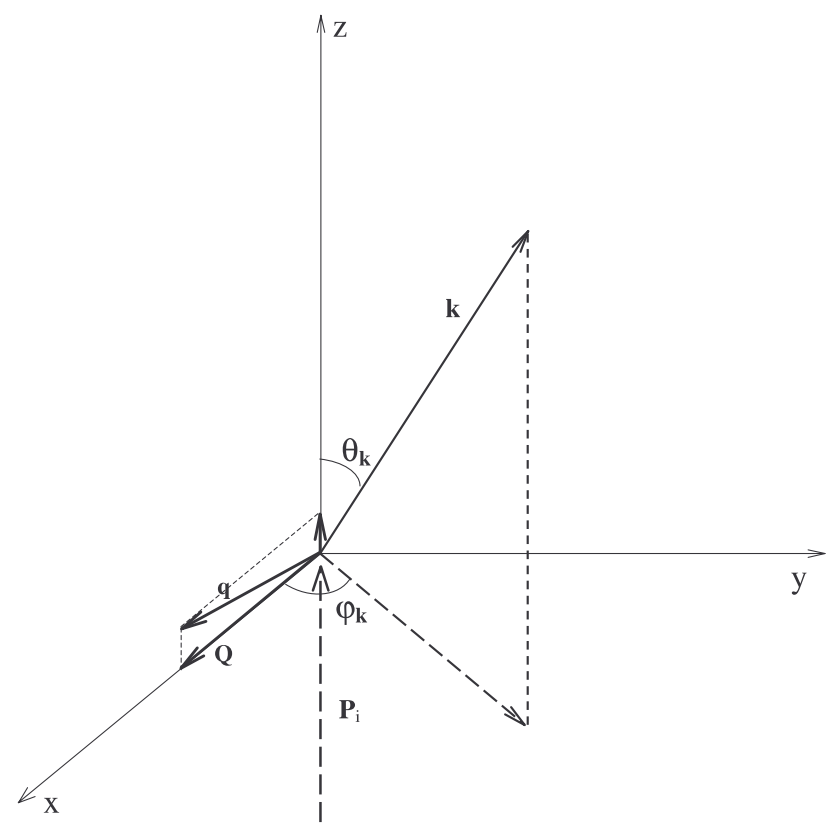

Figure 1

FIG. 1. Schematic illustration of the collision geometry. 


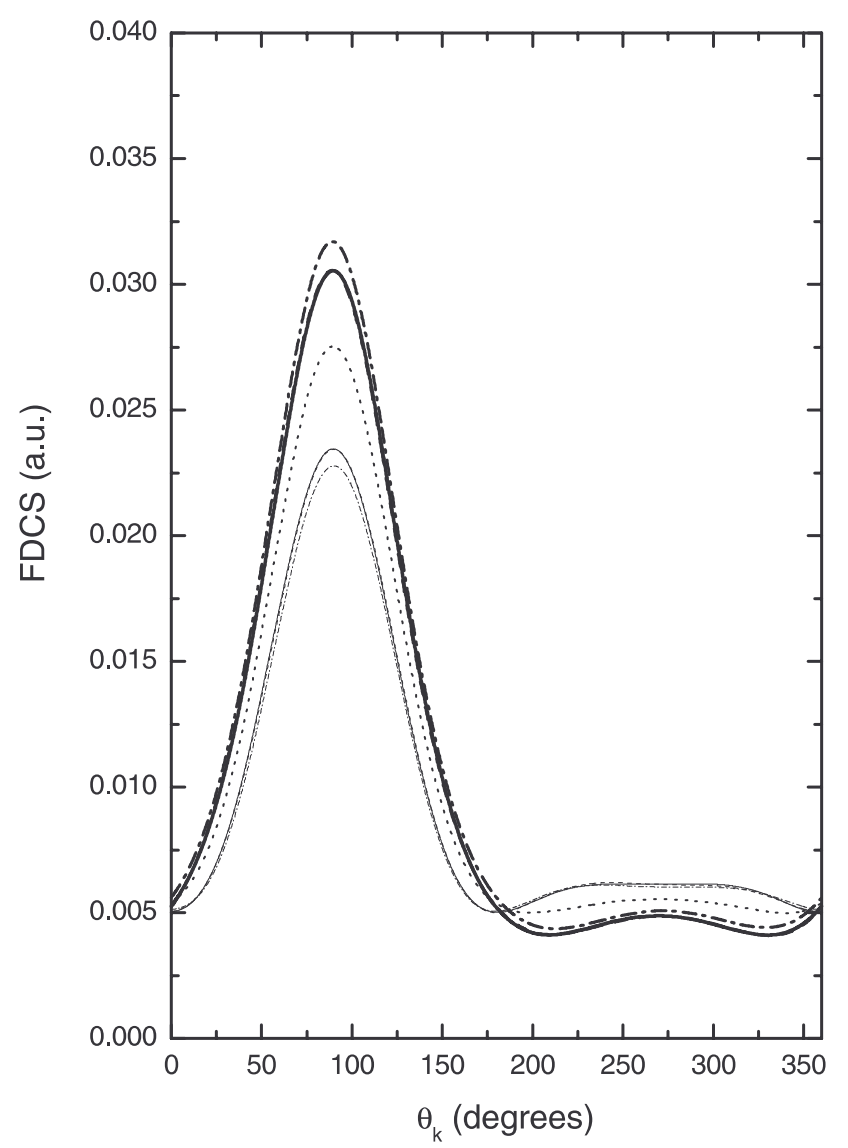

Figure 2

FIG. 2. Fully differential cross section (FDCS) in the collision plane $\left(\varphi_{k}=0^{0}\right)$ as a function of the polar emission angle $\vartheta_{k}$ for collisions with $100 \mathrm{MeV} / \mathrm{u} \mathrm{C}^{6+}$ and $\bar{C}^{6-}$. Electron emission energy $\varepsilon_{k}=1 \mathrm{eV}, Q=1$ a.u.. Dot curve: first order result; thick solid curve: Glauber result for $Z_{p}=6$; thin solid curve: Glauber result for $Z_{p}=-6$; thick dash curve: CDW-EIS result for $Z_{p}=6$; thin dash curve: CDW-EIS result for $Z_{p}=-6$; thick dash-dot curve: second order result for $Z_{p}=6$; thin dash-dot curve: second order result for $Z_{p}=-6$. Note that the Glauber and CDW-EIS results practically coincide in this case. 


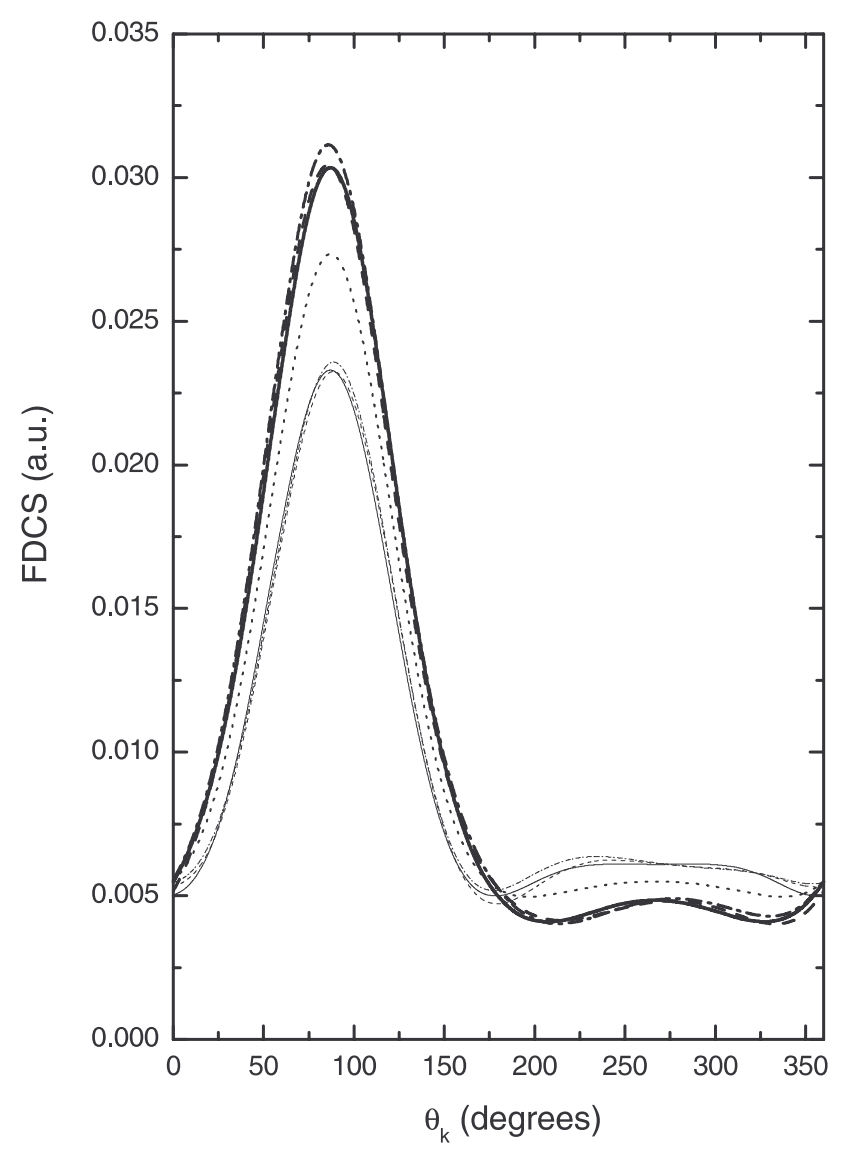

Figure 3

FIG. 3. Same as in figure 2 but for collisions with $2.5 \mathrm{MeV}$ protons and antiprotons. 


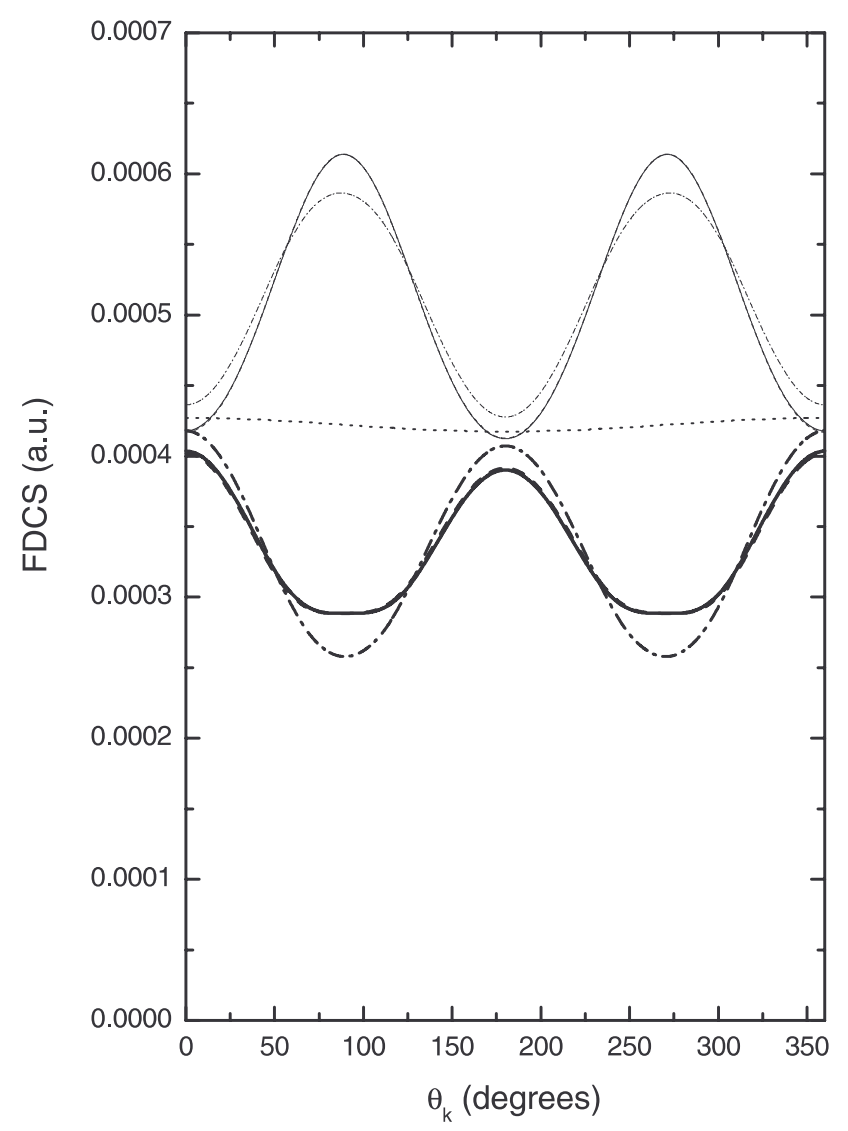

Figure 4

FIG. 4. FDCS in the plane $\varphi_{k}=90^{0}$ as a function of $\vartheta_{k}$ for collisions with $100 \mathrm{MeV} / \mathrm{u} \mathrm{C}^{6+}$ and $\bar{C}^{-6} \cdot \varepsilon_{k}=10 \mathrm{eV}, Q=1$ a.u.. Dot curve: first order result; thick solid curve: Glauber result for $Z_{p}=6$; thin solid curve: Glauber result for $Z_{p}=-6$; thick dash curve: CDW-EIS result for $Z_{p}=6$; thin dash curve: CDW-EIS result for $Z_{p}=-6$; thick dash-dot curve: second order result for $Z_{p}=6$; thin dash-dot curve: second order result for $Z_{p}=-6$. 


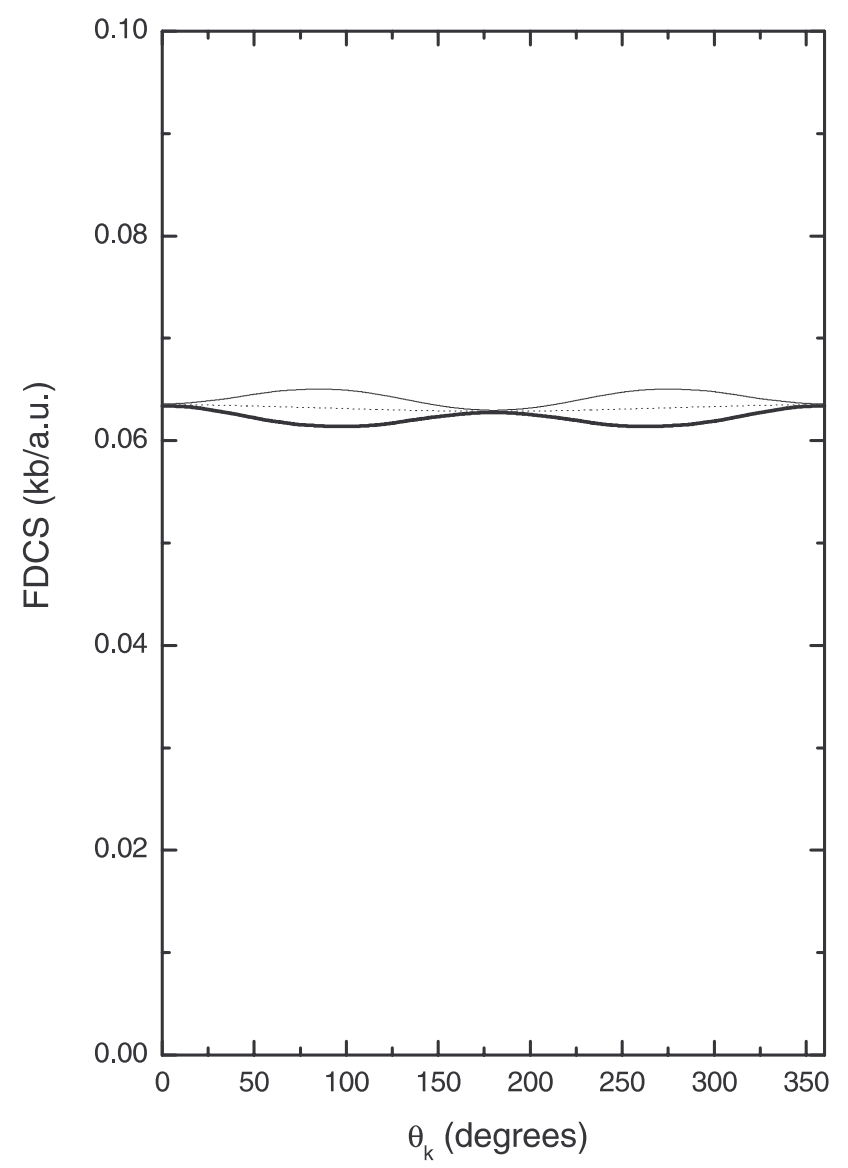

Figure 5

FIG. 5. Same as in figure 4 but for collisions with protons and antiprotons moving at a velocity very close to the speed of light, $v_{p}=137$ a.u. (only first order and Glauber results are shown). 


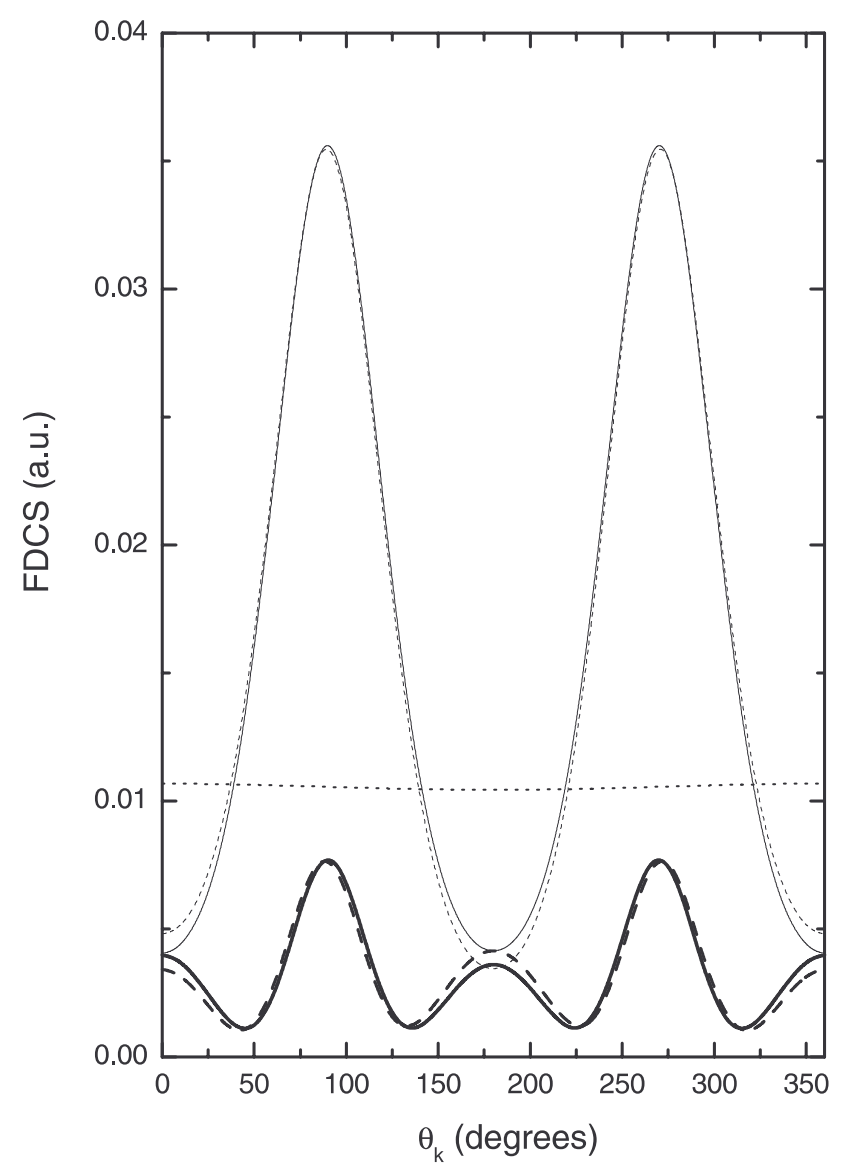

Figure 6

FIG. 6. FDCS in the plane $\varphi_{k}=90^{0}$ as a function of $\vartheta_{k} . \varepsilon_{k}=10 \mathrm{eV}, Q=1$ a.u., $v_{p}=60$ a.u., $Z_{p}= \pm 30$. Dot curve: first order result; thick solid curve: Glauber result for $Z_{p}=30$; thin solid curve: Glauber result for $Z_{p}=-30$; thick dash curve: CDW-EIS result for $Z_{p}=30$; thin dash curve: CDW-EIS result for $Z_{p}=-30$. 


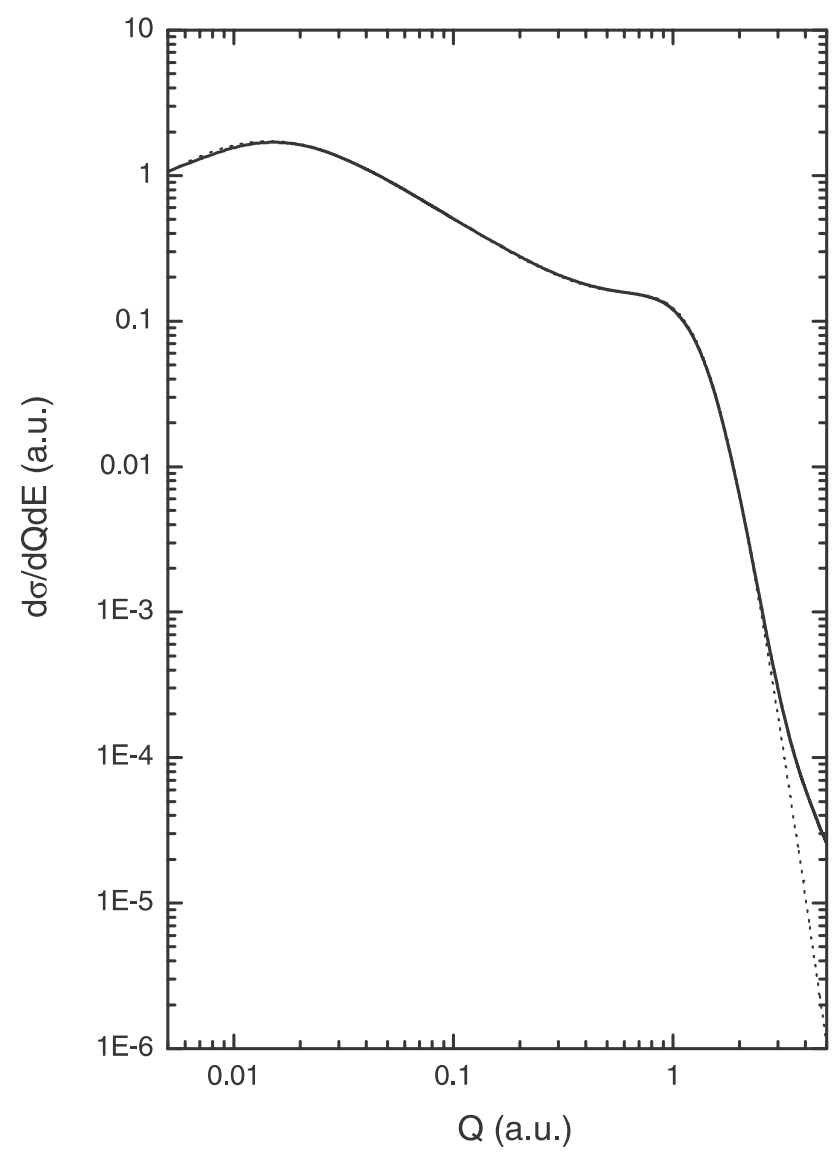

Figure 7

FIG. 7. Cross section $d^{2} \sigma / d \varepsilon_{k} d Q$ as a function of $Q$ at a fixed emission energy of $10 \mathrm{eV}$. Collisions with $100 \mathrm{MeV} / \mathrm{u} \mathrm{C}^{6+}$ ions. Solid curve: Glauber result; dot curve: first order result. CDW-EIS results (not shown) coincide with the Glauber ones. The shoulder at $Q \sim 1$ corresponds to the binary-encounter collisions in which the electron is emitted with momentum $k \simeq q$. 